\title{
UNIQUE PROPERTIES OF QUADRATIC SOLITONS
}

\author{
G.I. Stegeman, R. Fuerst, R. Malendevich, R. SchieK*, Y. Baeik ${ }^{\dagger}$ \\ School of Optics and CREOL, University of Central Florida \\ 4000 Central Florida Blvd., Orlando, FL 32816-2700, USA
}

I. BaumanN, W. SohLer

Angewandte Physik, Universität Paderborn

Warburger Strasse 100, 33098 Paderborn, Germany

G. Leo, G. Assanto

Dipartimento di Ingegneria Elettronica, Terza University of Rome

Via della Vasca Navale 84, 00146 Roma, Italy

Ch. Bosshard and P. Günter

ETH Honggerberg, Swiss Fed. Inst. of Tech., HPF, 8093 Zürich, Switzerland

Quadratic spatial solitons exist in media with second order nonlinearities near the phase-matching condition for frequency mixing processes involving two or three waves of different frequency. Discussed here are a number of properties of these special solitons which are different from those of other spatial solitons which rely on optically induced index changes for guiding. First, the self-guiding properties of quadratic solitons are shown to have completely different origins than solitons which rely on index changes. Second, it is shown that there exists a large variety of quadratic solitons which contain two or three distinct spectral components with relative amplitudes depending on the phase mismatch, dimensionality of the propagation geometry, the soliton power and the launching conditions. Third, under appropriate conditions, solitons can be formed even when the group velocity directions for the spectral components lead to walk-off under normal circumstances. Fourth, for type II phase-matching in bulk crystals, seeded interactions lead to saturating amplifier characteristics.

PACS numbers: 42.65.Tg, 42.65.Yj, 42.65.-k

*Permanent address: Electrophysik, TU München, ARCisstr. 21, 80333 München, Germany.

†Current address: Dept. of Physics, Washington State University, Pullman, WA 99164-2814; USA. 


\section{Introduction}

The period of last $15-20$ years has been an exciting time for solitons investigated experimentally in optics $[1,2]$. Temporal solitons have been developed to the point of demonstration of long haul, WDM communications over 10000 miles [3]. After a short lull, the field was revived recently by the concept of the "dispersion-managed" soliton and the practical application to long distance transmission seems likely again [4].

Spatial solitons, beams which do not spread in space (versus time for the temporal soliton), were recognized experimentally already in the 1970 s in media exhibiting saturating odd order nonlinearities $[2,5]$. However, these spatial analogs did not attract wide-spread attention until the mid to late 1980s when work on spatial solitons in glass, $\mathrm{CS}_{2}$, and finally in $\mathrm{AlGaAs}$ slab waveguides operated below half of the semiconductor band-gap was reported [2,6-8]. Nevertheless, the relevant nonlinearity was still $\chi^{(3)}$ in the slab waveguide demonstrations, the same as for temporal solitons in glass fibers. The only exception was the early experiment in a bulk medium in which self-trapping was possible due to higher order nonlinearities in addition to $\chi^{(3)}[5]$.

A large departure from these trends was reported in the early to mid 1990s. Photorefractive solitons were first reported in 1993, just a year after they were predicted theoretically $[2,9,10]$. This was soon followed by experimental reports of "quadratic solitons" which had already been predicted in the mid 1970s [11-13]. These solitons were unusual because the usual spatial diffraction is not arrested by a "self-focusing" nonlinear index change. Instead, the self-trapping mechanism is the rapid exchange of power between two or more waves of different frequency coupled together by the second order nonlinearity $\chi^{(2)}$.

In this paper we will discuss experiments on these quadratic solitons which illustrate some of the unique features not found with the other types of spatial solitons reported to date [2]. The next section deals with the simple coupled mode equations which describe quadratic solitons and their evolution for the $(1+1) \mathrm{D}$ case, i.e. in slab waveguides. A number of experiments relevant to this case are also discussed there. In the next section, the equations and experiments for the $(2+1) D$ geometry, i.e. bulk media will be presented. A discussion of some of the similarities and differences between quadratic solitons and other solitons will be given in the final section.

\section{2. $(1+1) D$ quadratic solitons}

\subsection{Equations governing the evolution and propagation of $(1+1) D$ quadratic solitons}

The coupled mode equations describing second harmonic generation for beams with finite cross-section in a slab waveguide are well known. They are sufficient for describing quadratic solitons. Consider a slab waveguide (1D) with propagation along the $z$-axis in the $y-z$ plane so that the guided wave confinement occurs along the $x$-axis. For the simplest case of type I phase-matching, the interacting fundamental and harmonic fields can be written, respectively, as 


$$
\begin{aligned}
& E_{1}(r, t)=(1 / 2) a_{1}(y, z) \exp \left[\mathrm{i}\left(\omega t-k_{1} z\right)\right]+\mathrm{cc}, \\
& E_{3}(r, t)=(1 / 2) a_{3}(y, z) \exp \left[\mathrm{i}\left(\omega t-k_{3} z\right)\right]+\mathrm{cc},
\end{aligned}
$$

where the subscript 1 identifies parameters at the fundamental frequency $\omega$, and the subscript 3 refers to the second harmonic $(2 \omega)$. (Later the subscript 2 will be used for the second, orthogonally polarized, input fundamental field in type II phase-matching.) The complex field amplitudes are $a_{i}(y, z)$. The corresponding coupled mode equations are

$$
\begin{aligned}
& \frac{\partial}{\partial z} a_{1}(y, z)-\frac{1}{2 \mathrm{i} k_{1}} \frac{\partial^{2}}{\partial y^{2}} a_{1}(y, z)=-\mathrm{i} \Gamma a_{1}^{*}(y, z) a_{3}(y, z) \exp (\mathrm{i} \Delta k z), \\
& \frac{\partial}{\partial z} a_{3}(y, z)+\rho_{3} \frac{\partial}{\partial y} a_{3}(y, z)-\frac{1}{2 \mathrm{i} k_{3}} \frac{\partial^{2}}{\partial y^{2}} a_{3}(y, z)=-\mathrm{i} \Gamma a_{1}^{2}(y, z) \exp (-\mathrm{i} \Delta k z) .
\end{aligned}
$$

Here $\Delta k=2 k_{1}-k_{3}$ is the linear wave vector mismatch ( $\Delta k L$ the phase-mismatch), $\Gamma$, the nonlinear coupling coefficient, is proportional to the effective second order susceptibility $\chi^{(2)}$ for the appropriate material symmetry class and field geometry, and $\rho_{3}$ is the harmonic Poynting vector walk-off angle from the $z$-axis. Here $\Gamma$ includes the "overlap integral" of the fundamental and harmonic guided wave fields. Note that walk-off between the two beams is absent for propagation along the principal optical axes of a crystal. However, in the more general case, walk-off can and does occur, complicating the soliton forming process in many doubling crystals. It is important to note that these equations describe all of the soliton phenomena discussed in this chapter, even the adiabatic (when valid) evolution from input launching conditions which do not correspond to the stable steady-state solutions. Missing is the coupling to radiation fields which occurs with highly non-ideal launching conditions (of the type actually used in some of the experiments described here). As a result the total launched power does not all end up in the solitons propagating along the $z$-axis. In general, the further away the launching conditions are from the final soliton "modes", the more non-adiabatic is the field evolution. For launching of the fundamental only at high input powers, an example of the evolution of the fields (at their peaks) is shown in Fig. 1. Note the oscillatory behavior that dies off with distance as the fundamental and harmonic fields readjust their relative amplitudes and phases to that of a quadratic soliton [14].

A key feature of quadratic solitons is that they consist of more than one field, component. In fact it is the power exchange between the fundamental and harmonic that leads to beam narrowing for both the fundamental and the harmonic, as illustrated in Fig. 2. The key is the form of Eq. (2). Clearly the driving term for the harmonic is proportional to the fundamental field profile squared, i.e. the driving term is narrower in space than the fundamental beam. As a result the initially generated harmonic is narrower than the fundamental. Ignoring spatial diffraction for the moment, the regenerated fundamental, proportional to $a_{1}^{*}(z) a_{3}(z)$, is also narrower than the initial fundamental. So, both beams are spatially narrowed by this exchange of power between the fundamental and harmonic. This narrowing mechanism compensates spatial diffraction, providing that enough power is converted to the harmonic and that the distance over which substantial conversion 


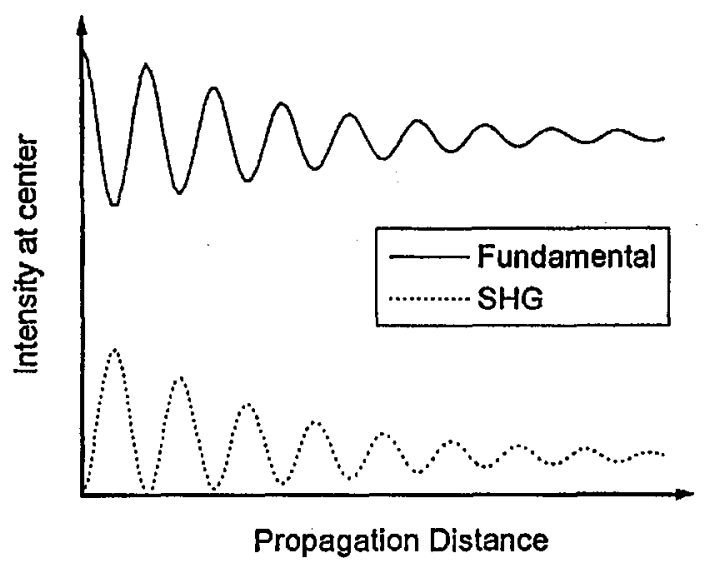

Fig. 1. Simulated spatial evolution towards a stable quadratic soliton of the peak fundamental and harmonic beam intensity when only the fundamental is excited at the input.

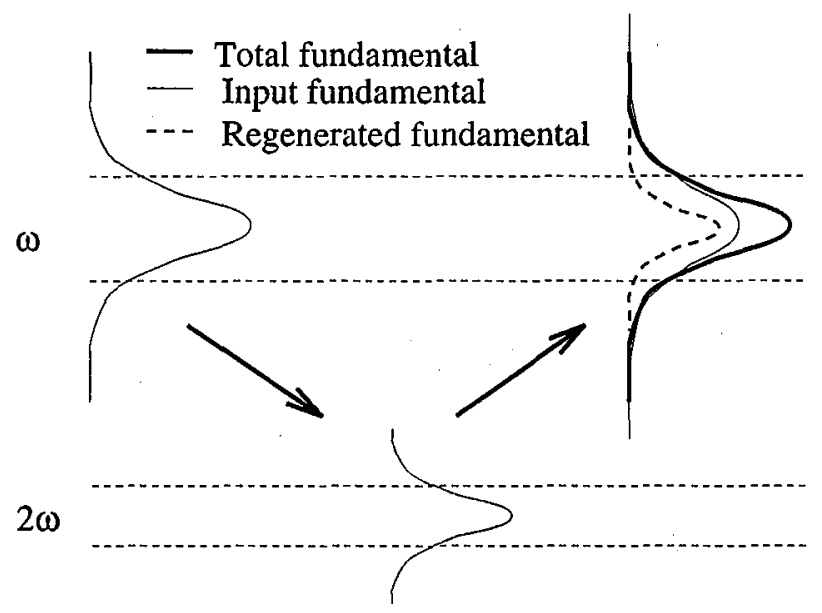

Fig. 2. Pictorial schematic showing the narrowing of the second harmonic relative to the fundamental, the narrowing of the regenerated fundamental, the diffracted fundamental which was not doubled and the total fundamental.

occurs, the parametric gain length, is of order of or smaller than the diffraction distance. If in addition there is walk-off of one or both beams, then the parametric gain length must also be shorter than the shortest walk-off length.

Another key difference between quadratic and other solitons is the composition of the soliton fields. The equilibrium value of the quadratic soliton components, their beam sizes and shapes depend on the wave vector mismatch as well as the total soliton power. An example of the calculated variation in the soliton threshold power and the relative fundamental and harmonic components are shown in Fig. 3 [15]: Note that the phase-mismatch is a key parameter in describing a quadratic soliton. 

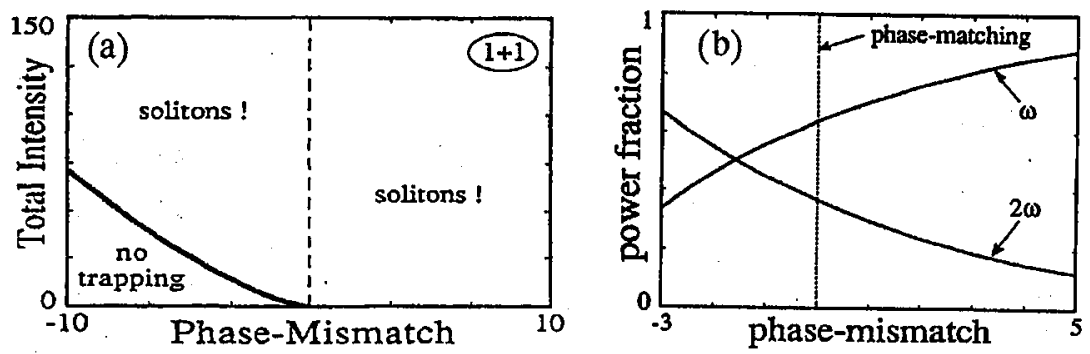

Fig. 3. (a) The total normalized soliton intensity versus the normalized phase mismatch for the type I, $(1+1) \mathrm{D}$ case. (b) The fraction of the soliton power in the fundamental and harmonic versus normalized phase mismatch for the type $\mathrm{I},(1+1) \mathrm{D}$ case.

\subsection{Quadratic soliton generation}

The only report to date of $(1+1) \mathrm{D}$ quadratic solitons has been in $\mathrm{LiNbO}_{3}$ Ti:indiffused waveguides [13]. The geometry is shown in Fig. 4. Note that when the beam is incident normal to the input face, both the fundamental and harmonic beams travel together along the $x$-axis which is an optic axis. However, for non-normal incidence in the $x-z$ plane, the group and phase velocities are collinear only for the $y$-polarized fundamental, but not for the $x-z$ polarized harmonic. Hence the group velocities are in slightly different directions, with the angular difference increasing with increasing input angle $\alpha$ from the input facet normal. When the quadratic soliton is formed, the fundamental and harmonics are so strongly coupled that they co-propagate, i.e. their group velocities are locked together and forced to be co-directional (and hence the phase velocities are no longer co-directional) [16]. The final soliton direction is governed by the relative fractions of the fundamental and harmonic powers which constitute the soliton, quite different from other spatial solitons which contain only one frequency component.

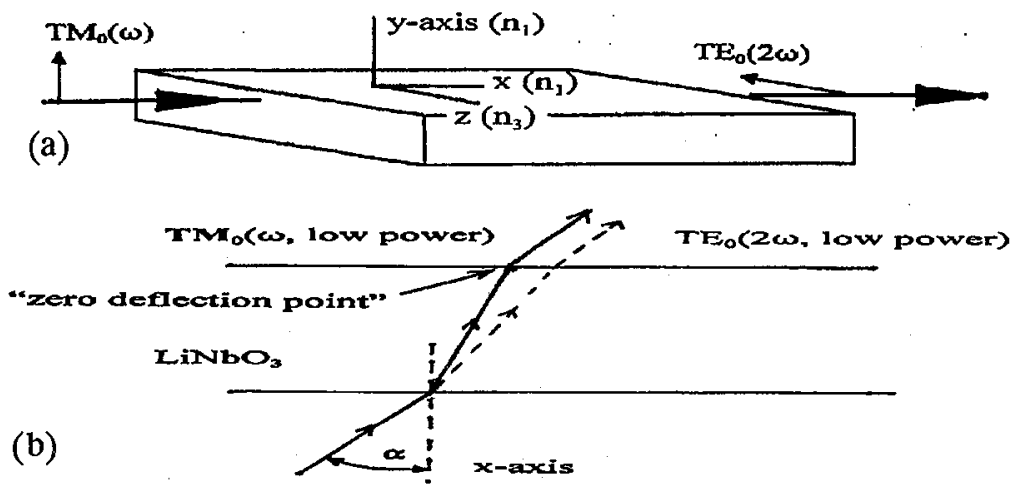

Fig. 4. (a) Lithium niobate waveguide geometry for the soliton experiments. The ordinary and extraordinary optic axes are $(x, y)$ and $z$, respectively. (b) Top view of the propagation of the uncoupled fundamental and harmonic beams through the waveguide, including walk-off. The "zero deflection point" is defined for Fig. 5. 


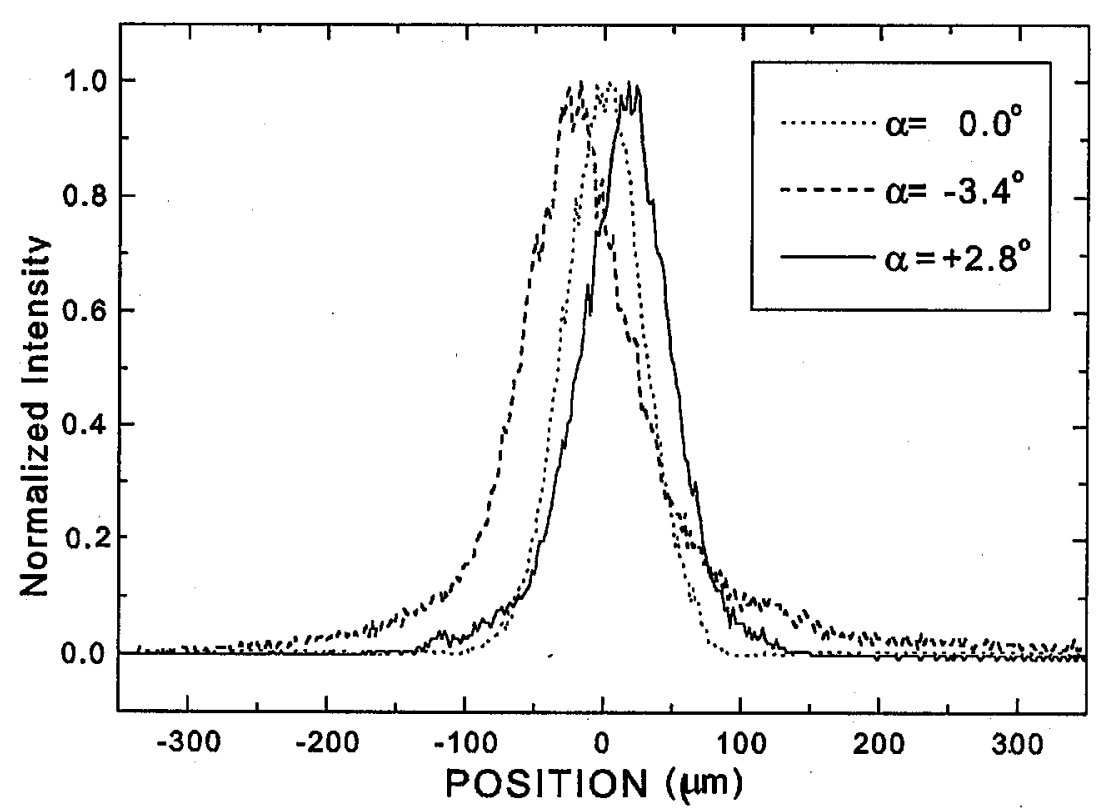

Fig. 5. The fundamental component of the soliton at the exit facet of the waveguide for three angles of incidence $\alpha$ in the $x-z$ plane from the normal to the input facet. "Zero" position is defined in Fig. 4.

Although quadratic solitons consist of both the fundamental and harmonic components, in our experiments we launched only the fundamental and rely on the generation of the required harmonic with distance into the sample. Hence it requires some propagation distance to establish the steady-state solitons. This distance typically increases when the input conditions are progressively further from those of a quadratic soliton. Note the example in Fig. 1.

The intensity profiles of the solitons observed at the output end of the sample are shown in Fig. 5 for various input angles $\alpha$ relative to the normal to the facet. The geometry of the measurement, shown in Fig. 4b, identifies the "zero" angle point in Fig. 5. Specifically, it is the angle at which a low power fundamental (non-solitonic) beam emerges from the end facet in the absence of soliton locking. So the horizontal axis is therefore a measure of the pulling of the fundamental propagation direction in the soliton away from that of a pure fundamental wave. The results are in excellent agreement with theory.

\subsection{Quadratic soliton collisions}

Because a quadratic soliton contains two components with different frequencies, one might expect that the interaction between two such solitons would exhibit unique behavior. However, the range of interaction and collision phenomena for quadratic solitons has been shown to be quite similar to that found in saturable media [17-20]. That is, depending on the crossing angle and relative phase between 


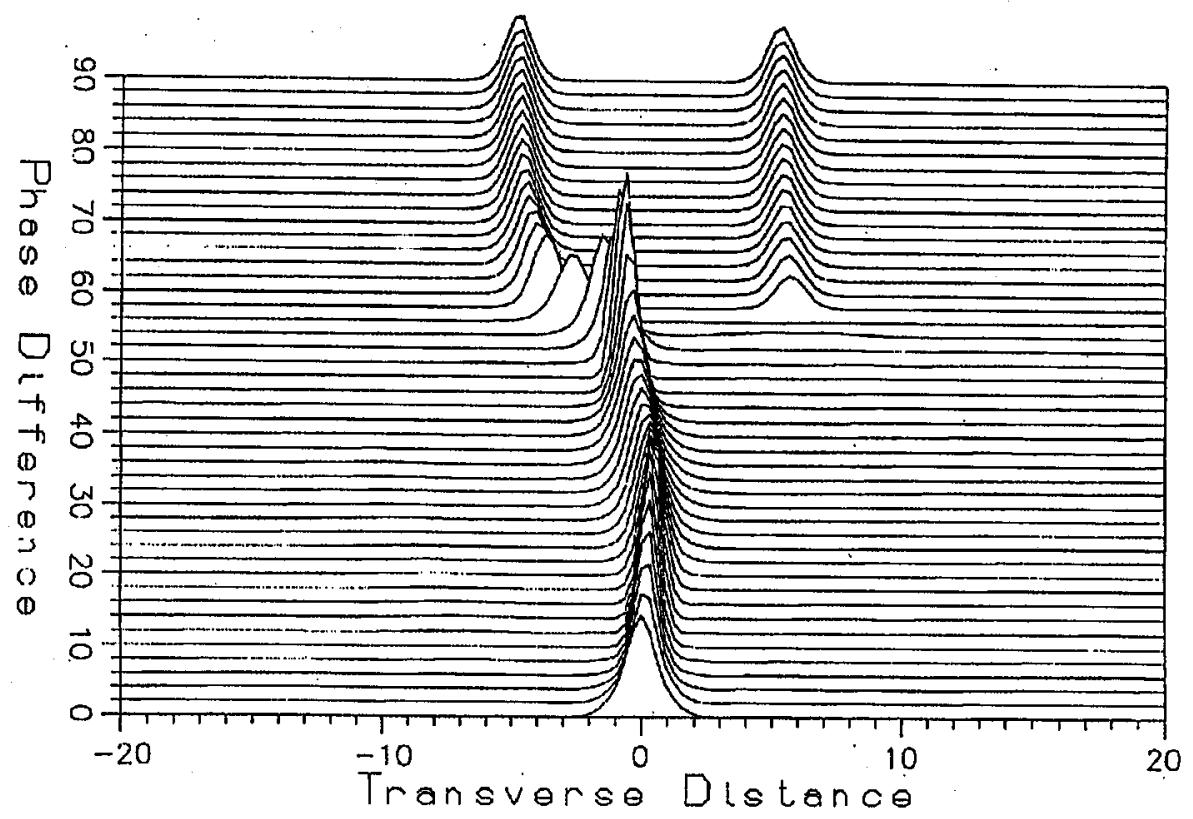

Fig. 6. The output from an interaction between two parallel input quadratic solitons after propagation for 20 diffraction lengths versus relative phase angle between the input solitons. Each horizontal slice corresponds to the results of a complete propagation.

the interacting solitons, the soliton either repel one another, attract one another, or exchange energy, and this has been verified experimentally.

There is, however, an interesting case uncovered numerically which has not been found to date in saturable media. As noted above, the same general phenomena still occur, but the transition from. one type of behavior to another exhibits interesting effects. There exists a sharp transition between fusion and repulsion with increasing phase angle difference between the input solitons for negative phase mismatch [18]. The numerical results are shown in Fig. 6 for $\beta=-3$, where $\beta=\Delta k k_{1} w_{0}^{2}$ is the normalized phase-mismatch and $w_{0}$ is the beam width. Here the output fields are shown after a propagation distance of 20 diffraction lengths as a function of the relative phase between two solitons launched parallel to one another at an initial separation of a few half-widths. Unfortunately, due to technical reasons it has not been possible to study this behavior in the negative phase-mismatch region of the $\mathrm{LiNbO}_{3}$ experiments discussed above.

\section{3. $(2+1) D$ quadratic solitons}

\subsection{Equations governing the evolution and propagation of $(2+1) D$ quadratic solitons}

The most general equations describing the harmonic generation process in the 2D, type II case are more complicated than for the previously discussed 1D, type I case. Here the extra fundamental quantities are identified with the subscript 2 and 


$$
\begin{aligned}
& \frac{\partial a_{1}}{\partial z}-\frac{1}{2 \mathrm{i} k_{1}}\left(\frac{\partial^{2} a_{1}}{\partial x^{2}}+\frac{\partial^{2} a_{1}}{\partial y^{2}}\right)=-\mathrm{i} \Gamma a_{2}^{*} a_{3} \exp (\mathrm{i} \Delta k z), \\
& \frac{\partial a_{2}}{\partial z}+\rho_{2} \frac{\partial a_{2}}{\partial x}-\frac{1}{2 \mathrm{i} k_{2}}\left(\frac{\partial^{2} a_{2}}{\partial x^{2}}+\frac{\partial^{2} a_{2}}{\partial y^{2}}\right)=-\mathrm{i} \Gamma a_{1}^{*} a_{3} \exp (\mathrm{i} \Delta k z), \\
& \frac{\partial a_{3}}{\partial z}+\rho_{3} \frac{\partial a_{3}}{\partial x}-\frac{1}{2 \mathrm{i} k_{3}}\left(\frac{\partial^{2} a_{3}}{\partial x^{2}}+\frac{\partial^{2} a_{3}}{\partial y^{2}}\right)=-2 \mathrm{i} \Gamma a_{1} a_{2} \exp (-\mathrm{i} \Delta k z),
\end{aligned}
$$

where $\Delta k=k_{1}+k_{2}-k_{3}$ and $\rho$ is the Poynting vector walk-off angle from the $z$-axis for launching along the $z$-axis. Although the interplay between the three different beams is complex, the net result is that each equation leads to beam narrowing and, as long as the parametric gain length is the shortest length in the problem, soliton locking still occurs.

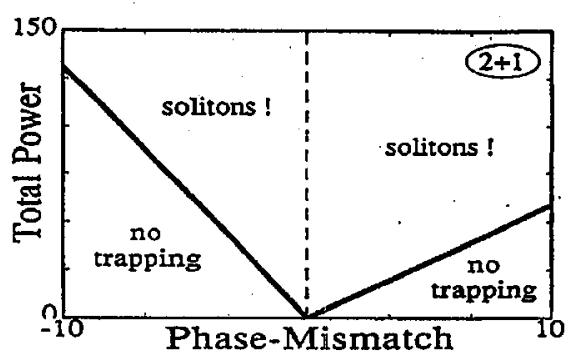

Fig. 7. The total normalized soliton power versus normalized phase mismatch for type I, $(2+1)$ D.
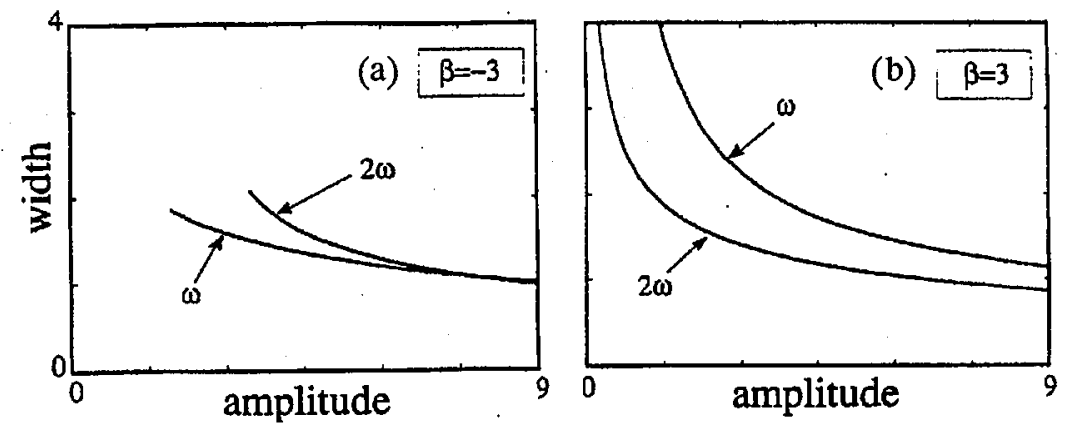

Fig. 8. The spatial width of the two spectral components versus their amplitudes for two different normalized phase mismatches; (a) $\beta=-3$, (b) $\beta=3$.

The type I case can easily be recovered from Eqs. (3) and is similar to the 1D case (Eqs. (2)) but with spatial diffraction in two dimensions. It is easier to discuss the salient features for the $(2+1) \mathrm{D}$ case using type I instead of type II. Shown in Fig. 7 is the trade-off between the soliton power and the normalized phase-mismatch [20]. In contrast to the $(1+1) \mathrm{D}$ case, a finite total power is needed to support quadratic solitons for both positive and negative phase mismatch. This result carries over to the type II geometry. Figure 8 illustrates the variation in the beam widths with amplitude [21]. Note that at high powers the asymptotic beam. sizes for the fundamental and harmonic converge towards the same value. 


\subsection{Type I quadratic soliton generation}

Potassium niobate is a well-known doubling crystal which yields non-critical type I, birefringent phase-matched doubling at a fundamental wa velength of $985 \mathrm{~nm}$ at $T=31.4^{\circ} \mathrm{C}$. The relevant nonlinearity is $d_{13}=-12 \mathrm{pm} / \mathrm{V}$.

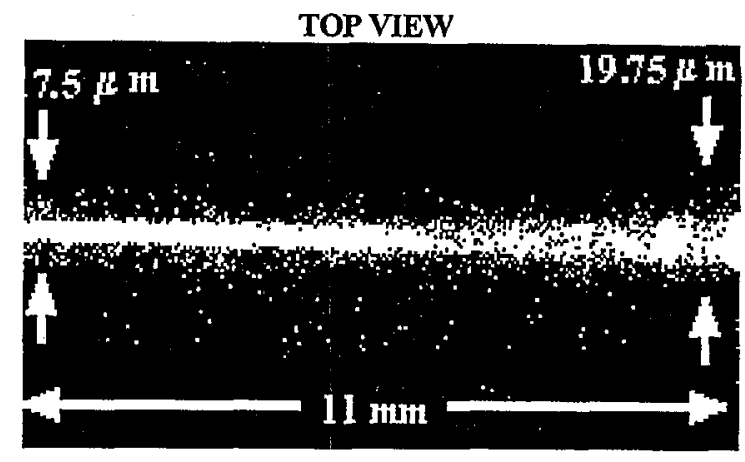

5 Diffraction Lengths

Fig. 9. A photograph of a quadratic soliton in bulk $\mathrm{KNbO}_{3}$ for type I SHG from a $985 \mathrm{~nm}$ fundamental input beam.

Shown in Fig. 9 for the first time is an actual photograph of a quadratic soliton propagating in a crystal with just a fundamental input, made possible by a very small concentration of defects and scattering centers within the crystal. Note the lack of diffraction spreading over a propagation distance of 5 diffraction lengths.

\subsection{Type II quadratic solitons}

For the type $\mathrm{II}$ experiments we used a standard KTP doubling crystal (for $1064 \mathrm{~nm}$ ) with $d_{\text {eff }}^{(2)}=2.7 \mathrm{pm} / \mathrm{V}, \rho_{2}=0.0033 \mathrm{rad}$ and $\rho_{3}=0.0049 \mathrm{rad}$ [12]. There are two orthogonally polarized fundamental beams, one of which is an o-ray and the other is an e-ray that propagates (eneigy) at an angle $\rho_{2}$ relative to the o-ray. The harmonic is also an o-ray so that at low powers all three beams walk away from each other on propagation. The diameter of the input beams was $20 \mu \mathrm{m}$, and they were focused onto the input face of the crystal. The beam output end-face of the crystal was imaged onto a camera. Again, only the fundamental beams were input into the crystal and the harmonic was generated on propagation.

Here we concentrate on two aspects of type II quadratic solitons which differ from the usual properties of spatial solitons based on odd order nonlinearities and index changes. The first is the extended family of solitons which exist for a wide range of ratios of intensities for the two fundamental polarizations, and the second is the amplifier-like effects which occur when the process is seeded.

\subsubsection{Extended family of spatial solitons}

In going from type I to type II another degree of freedom is introduced by the extra fundamental field $[22,14]$. This essentially allows quadratic solitons to be 
formed with a continuum of intensity ratios between the two fundamental beams. In practice only one fundamental beam is incident onto the crystal. It excites both an e- and o-polarized beam inside the crystal, with the ratio of e to o being determined by the angle of the incident polarization vector relative to the e-beam axis.

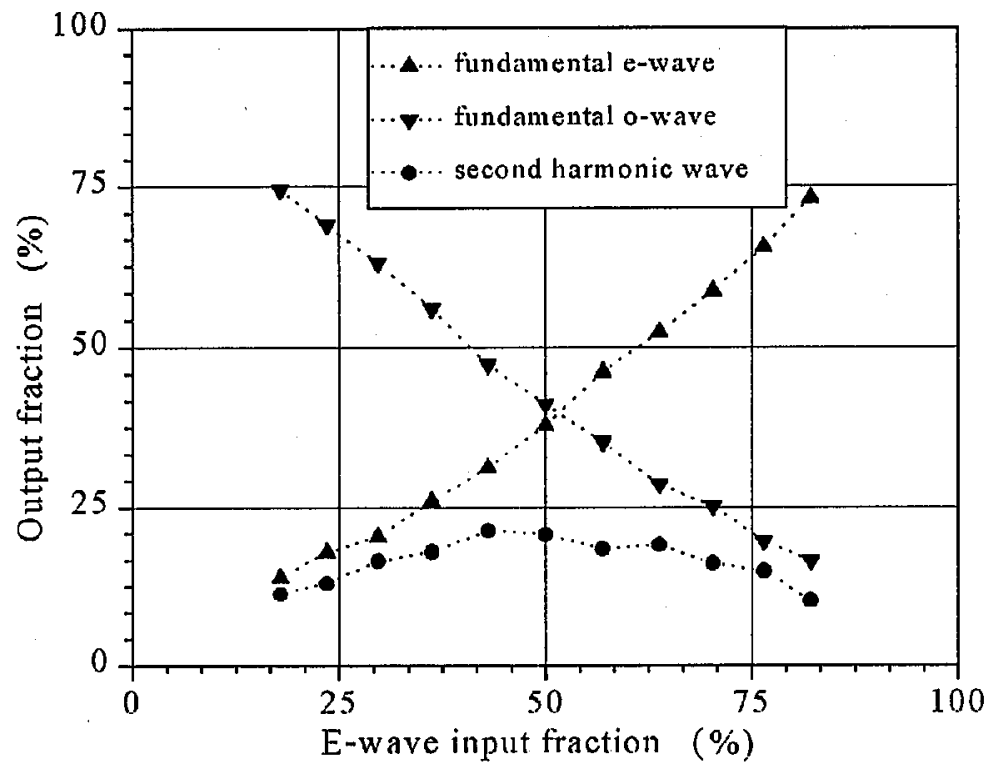

Fig. 10. Fraction of the output soliton in the e- and o-polarized fundamental waves, and the e-polarized second harmonic wave versus fraction of the input fundamental in the e-polarization. The process is on phase-match with an input fundamental energy of $13 \mu \mathrm{J}$.

The experimental results for the constituents of a soliton are shown in Fig. 10 for phase-matching [23]. The most obvious feature is that the e-beam component of the soliton is the largest one when the input fundamental has $I_{\mathrm{e}}>I_{\mathrm{o}}$, and vice versa. Over the range studied, ratios of almost $8: 1$ between the fundamental components were measured. Furthermore, the harmonic component is always the smallest with its peak value (which occurs when $I_{\mathrm{e}}=I_{\mathrm{o}}$ ) rising with decreasing phase-mismatch [23].

\subsubsection{Saturating amplification}

It is well known in second order nonlinear optics that a signal (seed) beam $\left(\omega_{\mathrm{s}}, k_{\mathrm{s}}\right)$ can be amplified by a powerful pump beam $\left(\omega_{\mathrm{p}}, k_{\mathrm{p}}\right)$ with the generation of an idler $\left(\omega_{\mathrm{i}}, k_{\mathrm{i}}\right)$, provided that energy and momentum are conserved, i.e. $\omega_{\mathrm{p}}=$ $\omega_{\mathrm{s}}+\omega_{\mathrm{i}}$ and $k_{\mathrm{p}}=k_{\mathrm{s}}+k_{\mathrm{i}}$, respectively. The salient question is whether quadratic solitons can be formed via amplification in this process.

The experiments were performed with the KTP crystal described above [24]. For a weak pump beam, only a diffracted pump beam is observed at the output. However, when the pump pulse $(0.53 \mu \mathrm{m})$ was sufficiently intense, in this case $10 \mathrm{~s}$ 
of $\mu \mathrm{J}$, the fundamental $(1.064 \mu \mathrm{m})$ was effectively seeded by noise and amplified to $\mu \mathrm{J}$ levels. These energy levels exceed those required for soliton locking and indeed a quadratic soliton was observed at the crystal output face. This is known as the degenerate case of an optical parametric generator. (The non-degenerate case, i.e. when $\omega_{\mathbf{s}} \neq \omega_{\mathbf{i}}$, has recently been reported by Trapani and coworkers [25].) However, for lower pump energies, of order $10 \mu \mathrm{J}$, a seed is required to trigger the amplification process. As shown in Fig. 11, only a few pJ's of seed are required to form the soliton. The most interesting feature there is the flat plateau for the fundamental output over 4-5 orders of magnitude of the input. This is the consequence of the formation of a quadratic soliton for which the intensity ratios of the harmonic to fundamental are fixed. Another interesting aspect of this process is that the amplification and soliton formation is independent of the polarization of the seed fundamental, another feature which is a consequence of soliton formation. This process could prove interesting for an amplifier in which a fixed output level is attractive, for example for digital logic.

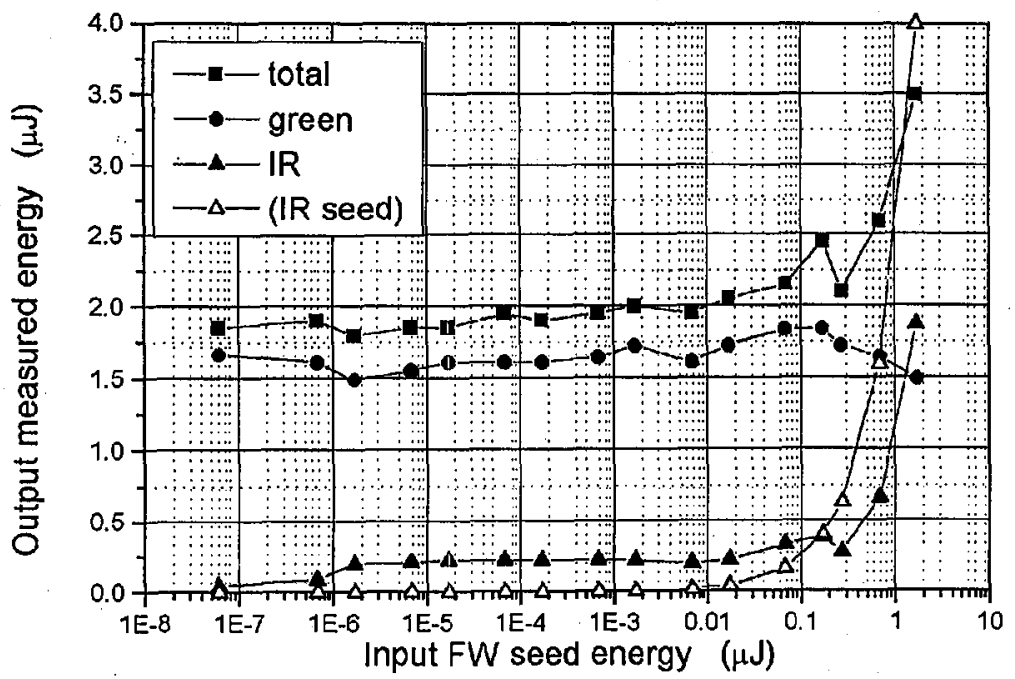

Fig. 11. The output energy for the harmonic (green) and fundamental (infrared) components of the soliton versus the fundamental seed beam energy. The pump energy was $8 \mu \mathrm{J}$.

An extension of this seeded process with a large cross-section pump beam and a small cross-section seed beam has recently been used to generate quadratic solitons nested inside the broad pump beam at the output [26].

\section{Summary}

Quadratic solitons exhibit many features uniquely different from those of other spatial solitons. These differences stem from both the multi-frequency nature of these solitons, and because the phase-mismatch is an additional free variable. For example, these two characteristics combine to produce a rich variety of 
solitons with different ratios of spectral components and polarization conditions. Especially rich are type II solitons in $(2+1) \mathrm{D}$ for which any ratio of the e- to o-beam fundamental polarizations leads to a stable quadratic soliton. These ratios can be further manipulated by changing the phase-matching condition $\Delta k L$, with the threshold for soliton formation increasing with increasing $|\Delta k L|$.

Another unique feature of quadratic solitons is that all of the soliton components propagate in the same group velocity direction, even though the individual waves would walk-off from each other in the absence of soliton formation. This is a direct consequence of the locking mechanism itself in which rapid power exchange guarantees the overlap of the interacting waves.

The gain inherent in second order processes also leads to a potentially useful amplifier. The key point is that (a) the phase-mismatch condition is fixed by geometry, (b) gain occurs with a weak fundamental seed with one pump photon yielding a photon in each of the fundamental polarizations, and therefore (c) the properties of the output solitons are fixed by (a), (b) and a fixed harmonic pulse energy as long as the seed energy is small compared to the final fundamental energy. Hence the output fundamental energy is independent of the input seed energy over 4-5 orders of magnitude of seed energy, i.e. the operational characteristics of a saturating amplifier.

\section{Acknowledgments}

This research was supported by NSF, BMDO, and AFOSR.

\section{References}

[1] A. Hasegawa, Optical Solitons in Fibers, Springer-Verlag, Berlin 1989.

[2] Spatial solitons reviewed extensively in: Opt. Quantum Electron. 30, 503 (1998).

[3] L.F. Mollenauer, P.V. Mamyshev, M.J. Neubelt, Electron. Lett. 32, 471 (1996).

[4] D.S. Govan, W. Forysiak, N.J. Doran, Opt. Lett. 23, 1523 (1998).

[5] J.E. Bjorkholm, A. Ashkin, Phys. Rev. Lett. 32, 129 (1974).

[6] J.S. Aitchison, A.M. Weiner, Y. Silberberg, M.K. Oliver, J.L. Jackel, D.E. Laird, E.M. Vogel, P.W.E. Smith, Opt. Lett. 15, 491 (1990).

[7] S. Maneuf, F. Reynaud, Opt. Commun. 66, 325 (1988).

[8] J.S. Aitchison, K. Al-Hemyari, C.N. Ironside, R.S. Grant, W. Sibbett, Electron. Lett. 28, 1879 (1992).

[9] M. Segev, B. Crosignani, A. Yariv, B. Fischer, Phys. Rev. Lett. 68, 923 (1992).

[10] G. Duree, J.L. Shultz, G. Salamo, M. Segev, A. Yariv, B. Crosignani, P. DiPorto, E. Sharp, R.R. Neurgaonkar, Phys. Rev. Lett. 71, 533 (1993).

[11] Yu.N. Karamzin, A.P. Sukhorukov, Zh. Eksp. Teor. Fiz. 68, 834 (1975) [Sov. Phys.JETP 41, 414 (1976)].

[12] W.E. Torruellas, Z. Wang, D.J. Hagan, E.W. VanStryland, G.I. Stegeman, L. Torner, C.R. Menyuk, Phys. Rev. Lett. 74, 5036 (1995).

[13] R. Schiek, Y. Baek, G.I. Stegeman, Phys. Rev. A 53, 1138 (1996).

[14] U. Peschel, C. Etrich, F. Lederer, B. Malomed, Phys. Rev. E 55, 7704 (1997).

[15] L. Torner, Opt. Commun. 114, 136 (1995). 
[16] L. Torner, D. Mazilu, D. Mihalache, Phys. Rev. Lett. 77, 2455 (1996).

[17] M.J. Werner, P.D. Drummond, J. Opt. Soc. Am. B 10, 2390 (1993).

[18] D.-M. Baboiu, G.I. Stegeman, L. Torner, Opt. Lett. 20, 2282 (1995).

[19] C. Etrich, U. Peschel, F. Lederer, B. Malomed, Phys. Rev. A 52, 3444 (1995).

[20] Y. Baek, R. Schiek, G.I. Stegeman, I. Baumann, W. Sohler, Opt. Lett. 22, 1550 (1997).

[21] L. Torner, D. Mihalache, D. Mazilu, E.M. Wright, W.E. Torruellas, G.I. Stegeman, Opt. Commun. 121, 149 (1995).

[22] A.V. Buryak, Y.S. Kivshar, Phys. Rev. Lett. 77, 5210 (1996).

[23] R.A. Fuerst, M.T.G. Canva, D. Baboiu, G.I. Stegeman, Opt. Lett. 22, 1748 (1997).

[24] M.T.G. Canva, R.A. Fuerst, D. Baboiu, G.I. Stegeman, G. Assanto, Opt. Lett. 22, 1683 (1997).

[25] P. Di Trapani, G. Valiulis, W. Chinaglia, A. Andreoni, Phys. Rev. Lett. 80, 265 (1998).

[26] P. Di Trapani, W. Chinaglia, Opt. Lett. 23, 1653 (1998). 